\title{
Influence of insulin and glucagon alone or combined on glucose and non-esterified fatty acids homeostasis in the lactating goat
}

\author{
P Schmidely, A Sleiman-Haidar, P Bas, A Rouzeau, D Sauvant \\ INRA, station de Nutrition et d'Alimentation de I'INA-PG, 16, rue Claude-Bernard, \\ 75231 Paris Cedex 05, France
}

Nutrients partitioning during lactation involves resistance to insulin (INS) (Grizard et al, 1988), whereas the role of glucagon (GLA) remains doubtful. As GLA and INS peak after ingestion of feed, the effects of INS and GLA alone or combined on glucose (GLU) and non-esterified fatty acids (NEFA) homeostasis were studied.

Twelve multiparous goats in mid-lactation (milk production $=3.1 \mathrm{~kg} / \mathrm{d}, \mathrm{SD}=0.7$ ) were injected after milking either with INS $(10 \mu \mathrm{g} / \mathrm{kg}, n=4)$, or GLA $(0.5 \mu \mathrm{g} / \mathrm{kg}, n=4)$ or a combination of INS + GLA $(10+0.5 \mu \mathrm{g} / \mathrm{kg}, n=4)$ after an overnight fast. Blood samples were collected before injection (IC) and 2, 6, 10, 14, 18, 22, 30, 46, 62 and $88 \mathrm{~min}$ after injection in order to measure GLU and NEFA levels.

Basal GLU was found to be $720 \mathrm{mg} / \mathrm{l}(\mathrm{SD}=$ 50 ) and basal NEFA $500 \mu \mathrm{EQ} /(\mathrm{SD}=50)$. INS decreased GLU to $40 \%$ of the IC value $30 \mathrm{~min}$ after injection (fig 1). Thereafter, GLU increased slowly but did not attain IC values. INS reduced NEFA during the first 14 min after injection, but this effect was then reversed and NEFA rose to $245 \%$ of IC values at $46 \mathrm{~min}$. This lipomobilisation could provide NEFA for oxydation and glycerol for neoglucogenesis. GLA was found to have a neoglucogenic effect as it increased GLU to $225 \%$. It simultaneously decreased NEFA to $60 \%$ of the IC value, at least partly because of the increase in INS concentration (results not shown). After this, NEFA increased but to a lesser extent than with INS alone. After GLA + INS injection, the short-term variations in GLU and NEFA were found to be fairly similar to the response with GLA alone. However, although the GLU peak was as high as with GLA alone it decreased more rapidly between 14 and $42 \mathrm{~min}$ to reach $46 \%$ of IC at $62 \mathrm{~min}$, probably because of a more elevated INS concentration (results not shown). The NEFA began to rise after $30 \mathrm{~min}$.

In conclusion, the main consequence of simultaneously injecting high doses of INS and GLA shows a prioritary effect of GLA followed by a delayed effect of INS. With such hormonal doses, mechanisms (probably hormone-dependent) which cause lipolysis in adipose tissues are initiated to compensate for the glucose shortage.

Grizard J, Champredon C, Aina E, Sornet C, Debras $E$ (1988) Hormon Metab Res 20, 15-22
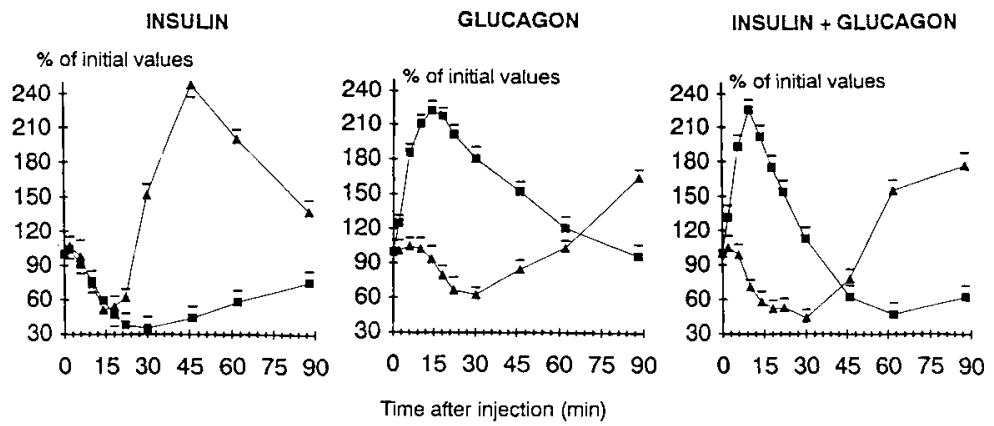

Fig 1. Plasma glucose $(\overline{\boldsymbol{\square}})$ and NEFA $(\overline{\boldsymbol{\Delta}})$ after hormone injection. 